\title{
Multiboson production measurements at the CMS experiment
}

\section{Riccardo Bellan* on behalf of the CMS collaboration}

Università degli studi di Torino and INFN

E-mail: riccardo.bellanecern.ch

Precision measurements of multiboson production are a validation of the Standard Model. These multiboson processes are a background to Higgs boson measurements and searches for Beyond the Standard Model physics. In this contribution, we presented recent measurements of multiboson final states performed in CMS, involving $\mathrm{W}, \mathrm{Z}$ and photon combinations. Inclusive and differential cross sections were compared to different theoretical predictions. Phase space regions that provide sensitivity to anomalous triple or quartic gauge couplings were also shown. These coupling strengths are directly related to the broken electroweak symmetry and deviations from the SM are a clear signal of new physics.

The 39th International Conference on High Energy Physics (ICHEP2018)

4-11 July, 2018

Seoul, Korea

${ }^{*}$ Speaker. 
The Standard Model (SM) of particle physics has been proven to be a theory capable of modeling with high accuracy the interaction among particles. At present, very few corners of the SM are yet unexplored. Among those is the detailed understanding of the Electroweak Symmetry Breaking (EWSB) mechanism, whose comprehension passes through the study of the vector boson productions. In this contribution we presented the latest CMS results about multiboson production studies. The description of the CMS detector can be found in ref. [1].

The study of the $\mathrm{pp} \rightarrow \mathrm{ZZ} \rightarrow 4 \ell$ differential cross sections [2] made a significant progress, extending the measurements to the associate production with jets [3]. The measurements start to be able to discriminate between next-to-leading-order (NLO) and next-to-next-to-leading-order (NNLO) in QCD predictions. The introduction of electroweak NLO corrections is desired.

The new measurement of the $\mathrm{pp} \rightarrow \mathrm{WZ} \rightarrow 3 \ell v$ total and differential cross-sections at $\sqrt{s}=$ $13 \mathrm{TeV}$ [4] has also been presented. $\mathrm{W}^{+} \mathrm{Z}$ and $\mathrm{W}^{-} \mathrm{Z}$ production cross section asymmetry has been measured as well, and found in agreement with theoretical calculation.

Searches for $\mathrm{pp} \rightarrow \mathrm{W} \gamma \gamma \rightarrow \ell v \gamma \gamma$ and $\mathrm{pp} \rightarrow \mathrm{Z} \gamma \gamma \rightarrow 2 \ell \gamma \gamma$ in $8 \mathrm{TeV}$ collision data [5] have been reported. The latter process has been observed with a significance close to 6 standard deviations.

Overall, the CMS Collaboration explored a wide set of processes involving the production of diboson and triboson intermediate states, whose summary can be found in Fig. 1.

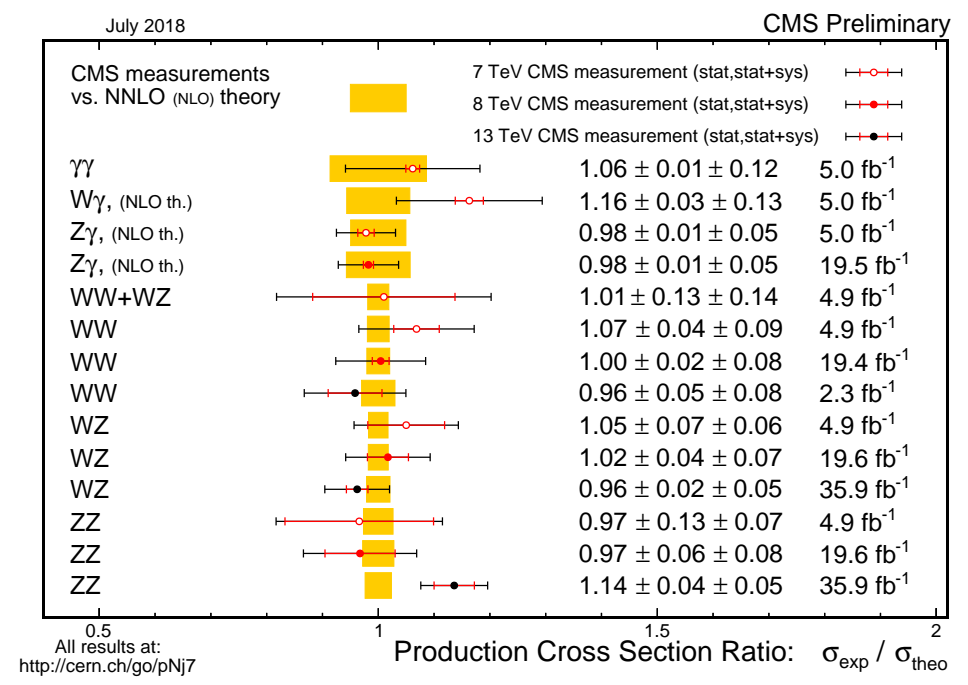

Figure 1: Summary of the diboson production cross sections measured by the CMS Collaboration [7].

Along with the Standard Model measurements, anomalous gauge couplings have been tested, without finding any deviation from pure SM predictions, even in analyses designed to maximize the sensitivity to anomalous couplings, such as $\mathrm{pp} \rightarrow \mathrm{WW} / \mathrm{Z} \rightarrow \ell v q q^{\prime}$ [6]. Figures 2 and 3 report a summary of the limits obtained by the CMS Collaboration. The limits on anomalous couplings are now more stringent than those from previous collider experiments.

Details on multiboson production studies can be found on the public pages of the CMS experiment [7]. 


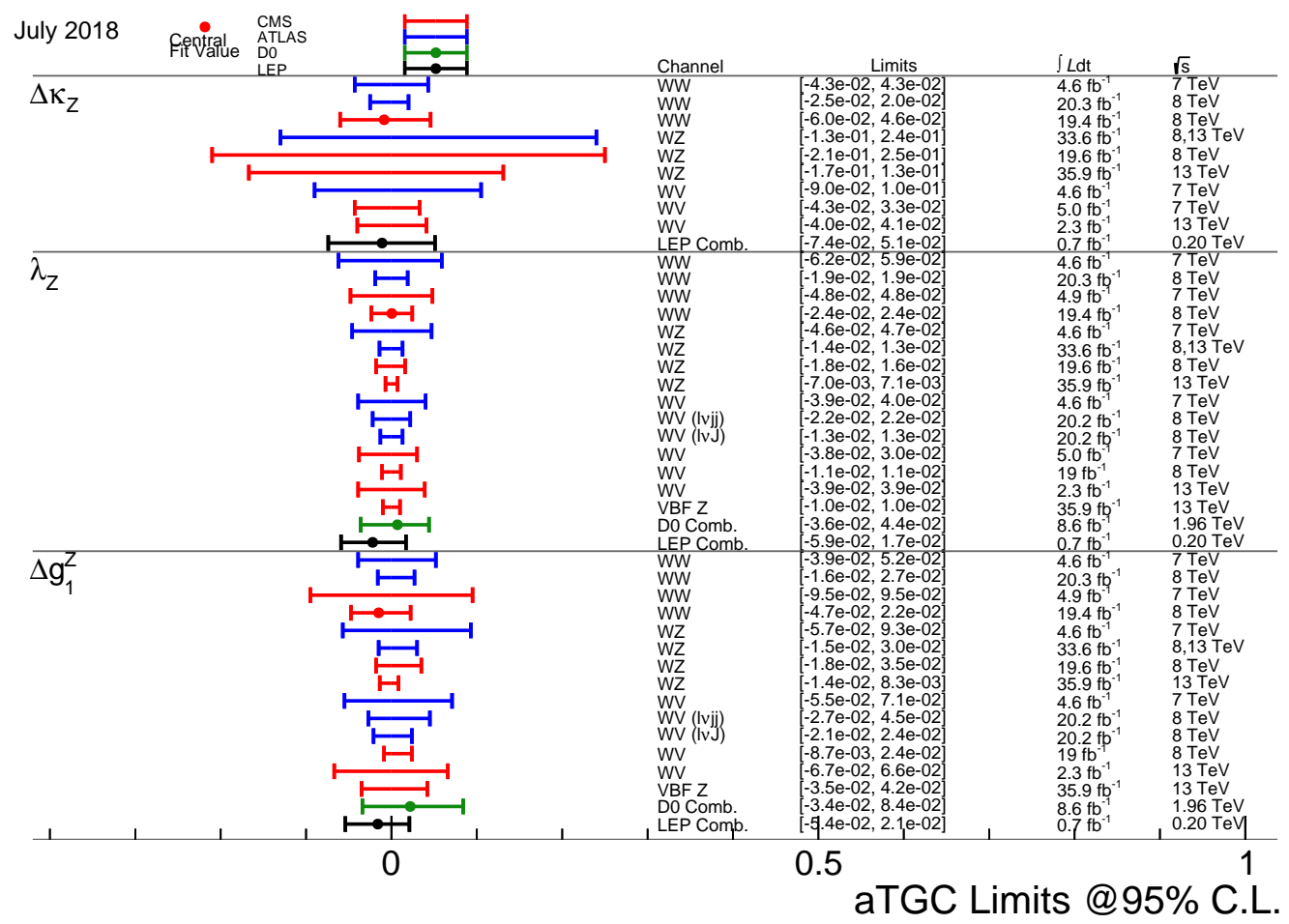

Figure 2: Summary of the investigation of possible anomalies in the WWZ triple gauge couplings in the electroweak sector [7].

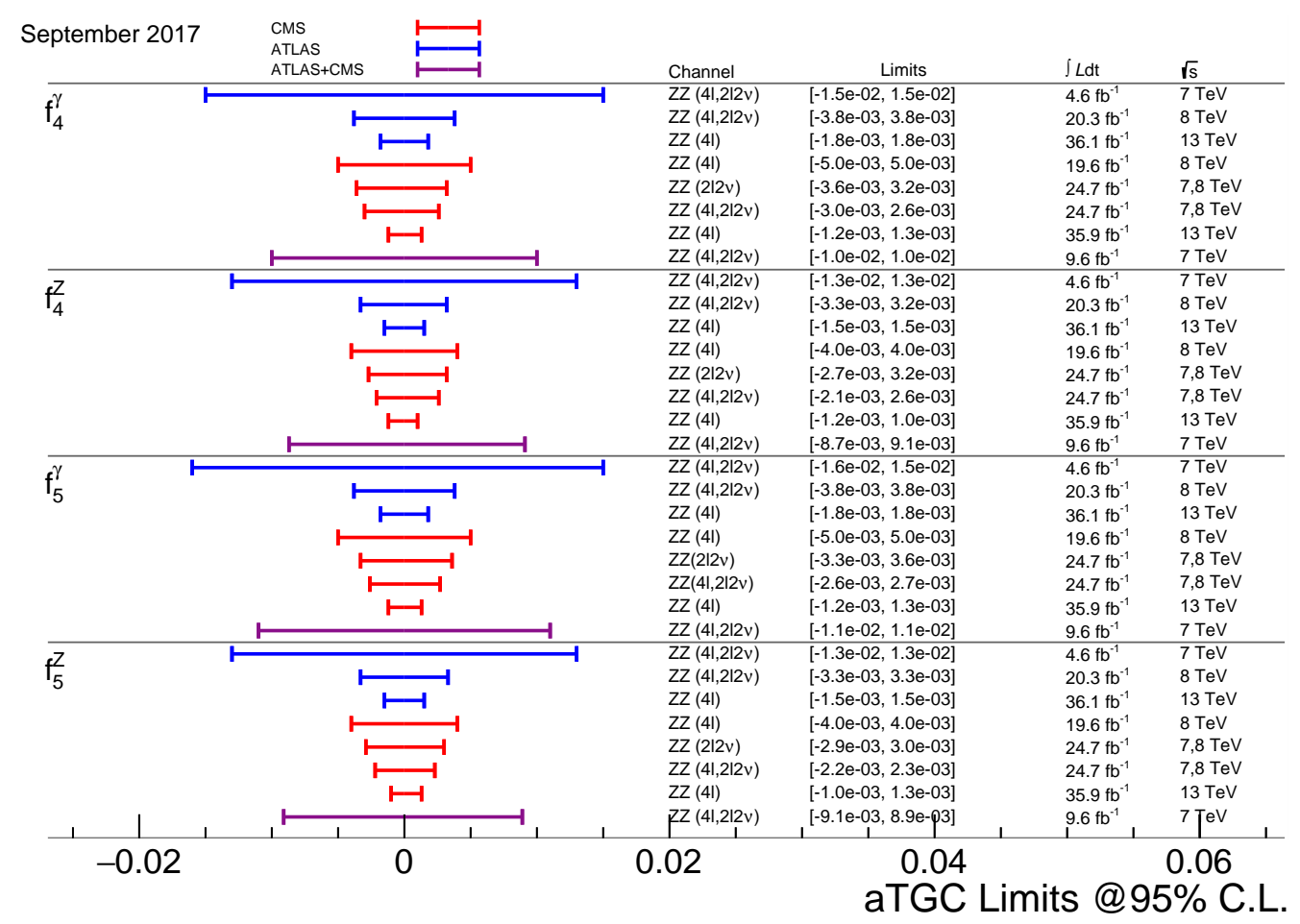

Figure 3: Summary of the investigation of possible anomalies in the $\mathrm{ZZ} \gamma$ and $\mathrm{ZZZ}$ triple gauge couplings in the electroweak sector [7]. 


\section{References}

[1] CMS Collaboration, The CMS experiment at the CERN LHC, JINST 3 S08004 (2008).

[2] CMS Collaboration, Measurement of the $\mathrm{pp} \rightarrow \mathrm{ZZ}$ production cross section, $\mathrm{Z} \rightarrow 41$ branching fraction and constraints on anomalous triple gauge couplings at $\sqrt{s}=13 \mathrm{TeV}$, arXiv:1709.08601, Eur. Phys. J. C 78 (2018) 165.

[3] CMS Collaboration, Measurement of differential cross sections for $\mathrm{Z}$ boson pair production in association with jets at $\sqrt{s}=8$ and $13 \mathrm{TeV}$, arXiv:1806.11073, accepted by Phys. Lett. B.

[4] CMS Collaboration, Measurements of the $\mathrm{pp} \rightarrow \mathrm{WZ}$ inclusive and differential production cross section and constraints on charged anomalous triple gauge couplings at $\sqrt{s}=13 \mathrm{TeV}$, CMS PAS 18-002, https://cds.cern.ch/record/2628761.

[5] CMS Collaboration, Measurements of the $\mathrm{pp} \rightarrow \mathrm{W} \gamma \gamma$ and $\mathrm{pp} \rightarrow \mathrm{Z} \gamma \gamma$ cross sections and limits on anomalous quartic gauge couplings at $\sqrt{s}=13 \mathrm{TeV}$, arXiv:1704.00366, JHEP 10 (2017) 072.

[6] CMS Collaboration, Search for anomalous couplings in semileptonic WW and WZ decays at $\sqrt{s}=13 \mathrm{TeV}$, CMS PAS 16-012, https://cds.cern.ch/record/2209148.

[7] CMS Standard Model Physics public web page, http://cms-results.web.cern.ch/cms-results/public-results/publications/SMP/index.html. 